D. S. Mathewson and V. L. Ford Mount Stromlo and Siding Spring Observatories The Australian National University

\title{
THE MAGELLANIC SYSTEM
}

The global distribution of $\mathrm{HI}$ in the Magellanic System is shown in Figure 1. The gas covers some 1500 square degrees of sky and has a mass of $1.8 \times 10^{9} \mathrm{M}_{\mathrm{O}}$. There are four main components: the LMC, the SMC, the inter-Cloud region and the Magellanic Stream. The integrated HI of the first three components is mapped in Figure 2 with the Parkes 64-m radio telescope which has a resolution of 15 arc min. The previous surveys of McGee and Milton (1966), Hindman (1967), Mathewson et al. (1979) have been combined with a recent survey by Mathewson et al. (1983) of the outer regions of the System to give this large-scale picture of the gas distribution. The last two surveys were made with a velocity resolution of $4.12 \mathrm{~km} \mathrm{~s}^{-1}$ and a minimum detectable signal of $0.2 \mathrm{~K}$. The long spurs extending from the LMC and SMC and the bridge joining the two galaxies with prominent spurs pointing to the Magellanic stream are all compelling evidence for tidal interaction between the LMC and SMC (Mathewson 1976a, Murai and Fujimoto 1980). The detailed velocity field of the HI is given in Mathewson et al. (1983). Its large-scale features are shown in Figure 5 of Mathewson et al. (1979) which indicate that the radial velocities of the inter-Cloud region form a velocity continuum with those of the LMC and SMC. This plus the continuity of the general velocity gradient across the entire Magellanic System strongly suggest that the two galaxies are bound.

\section{THE LARGE MAGELLANIC CLOUD}

The radial velocity field of the LMC is shown in Figure 3 from observations with the 18-m Parkes radio telescope. The effect of a translational motion of $250 \mathrm{~km} \mathrm{~s}^{-1}$ of the LMC along the great circle of the Magellanic Stream in the direction of the galactic plane has been removed from the observed velocities (Mathewson et al. 1977; Feitzinger et al. 1977; Lin and Lynden-Bell 1982). This has the effect of moderating the anomalously high velocities observed along the eastern edge of the LMC. The velocity field is peculiar and it is clear that 


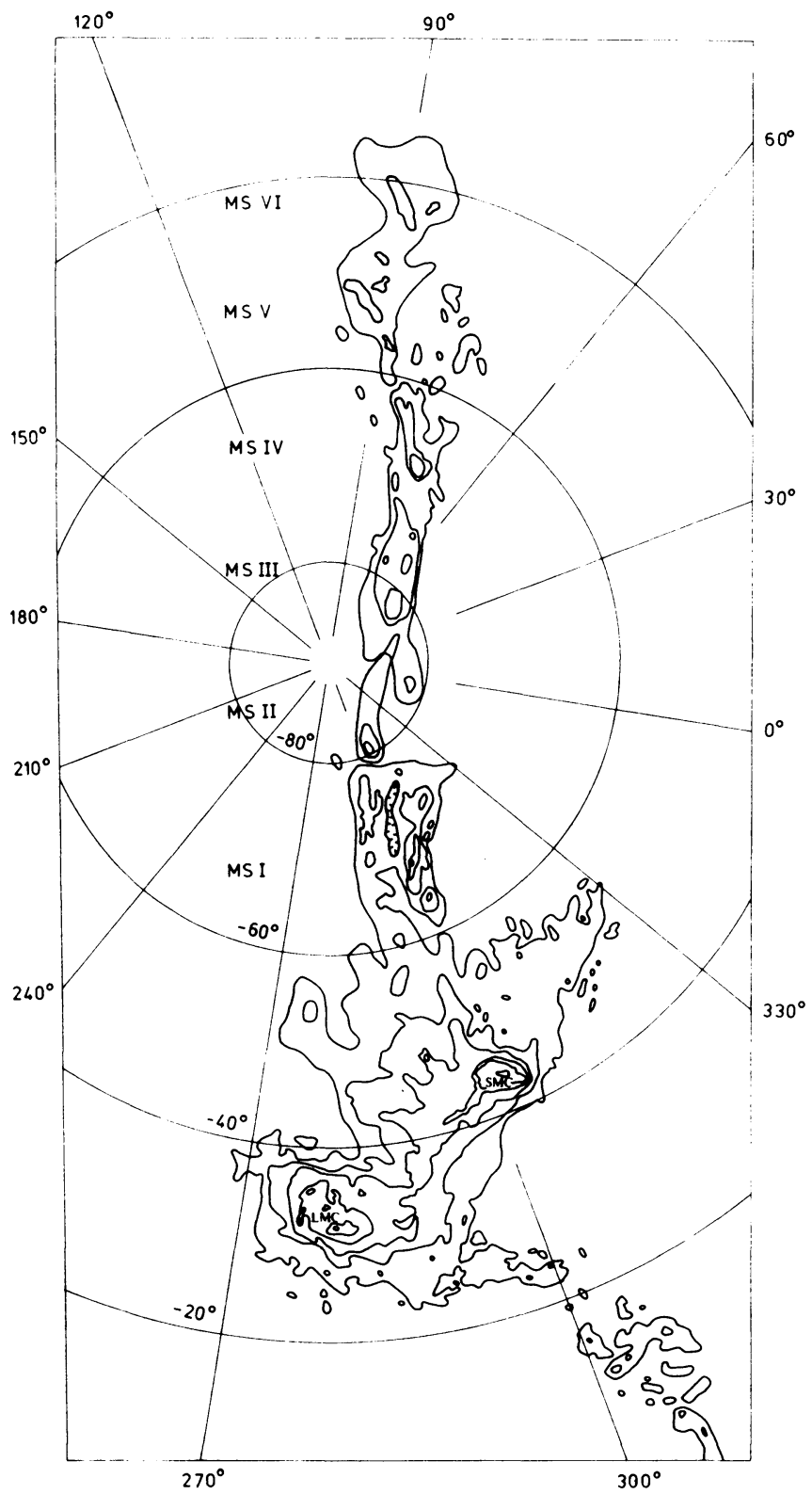

Fig. 1 The global distribution of HI in the Magellanic System in galactic co-ordinates. The outer contour is the $10^{19}$ atoms $\mathrm{cm}^{-2}$ isophote and the inner contours have been selected to show the main features of the components. The Magellanic stream is divided into six HI concentrations, MSI-VI, (Mathewson et al. 1977). Data have been taken from Mathewson (1976b), Mathewson et al. (1979), Mathewson, Ford and Fisher (1983), McGee and Milton (1966), Hindman (1967), Wannier and Wrixon (1972), Haynes, (1979), Mirabel (1981), Cohen (1982), and Morras (1983). 


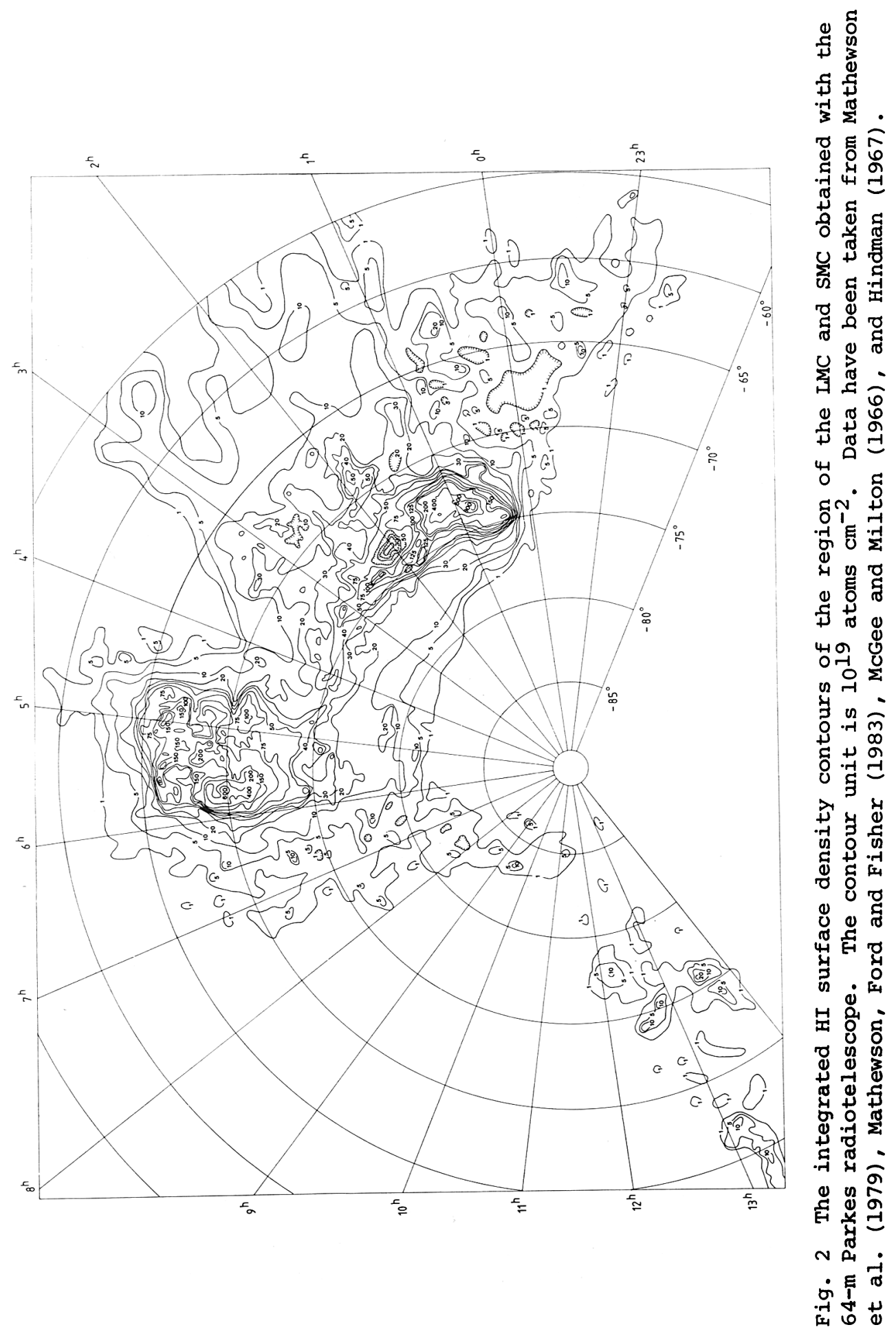




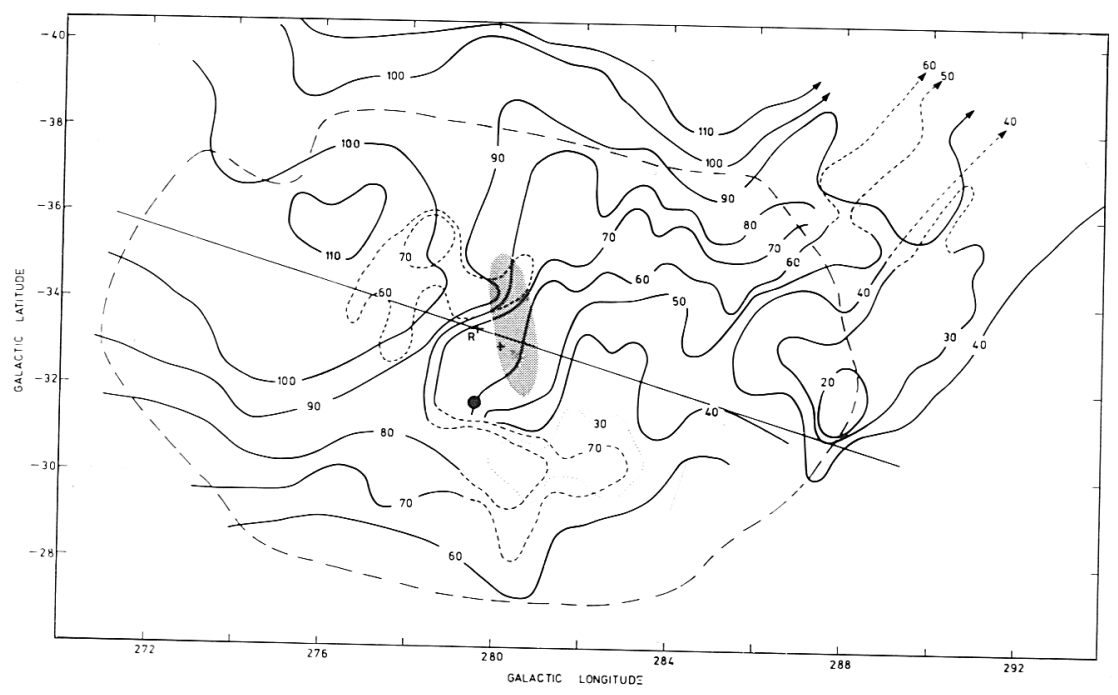

Fig 3 The radial velocity field of the LMC in galactic co-ordinates obtained using the 18-m Parkes radiotelescope. The contour numbers are in $\mathrm{km} \mathrm{s} \mathrm{s}^{-1}$ and represent the $\mathrm{V}_{\mathrm{GSR}}$ of the HI peaks (calculated using $\mathrm{V}_{\odot}=225 \mathrm{~km} \mathrm{~s}^{-1}$ ). The dotted contours indicate regions where 2 peaks occur in the HI profile. The effect of a translational motion of $250 \mathrm{~km} \mathrm{~s}^{-1}$ of the Magellanic Clouds has been removed. The dot is 30 Doradus and the shaded region is the Bar. The dashed line is the outer limit of the LMC clusters.

tidal interaction between the LMC and SMC has produced substantial warping of the plane of the LMC, complex $z$ structure and non-circular motions (Feitzinger 1980; Rohlfs et al. 1984). The velocity field of the LMC is similar in many respects to M83 which Rogstad et al. (1974) modelled successfully by a series of rings having a progression in their angle of inclination and position angle of the line of nodes with increasing radii.

\section{THE SMALL MAGELLANIC CLOUD}

A well-known characteristic of the SMC HI profiles is their double peaks separated by 30-40 $\mathrm{km} \mathrm{s}^{-1}$ (Hindman 1967; McGee and Newton 1981; Bajaja and Loiseau 1982). Hindman proposed that this velocity structure originates in three expanding shells of gas. This explanation is untenable as the double peaks occur throughout the SMC and are not confined to the areas covered by Hindman's shells. Figure 4 maps the velocity structure along the major axis of the SMC obtained with the 64-m reflector. This clearly shows the splitting of the gas into two components with similar velocity gradients of about $8 \mathrm{~km} \mathrm{~s}^{-1}$ per degree. 


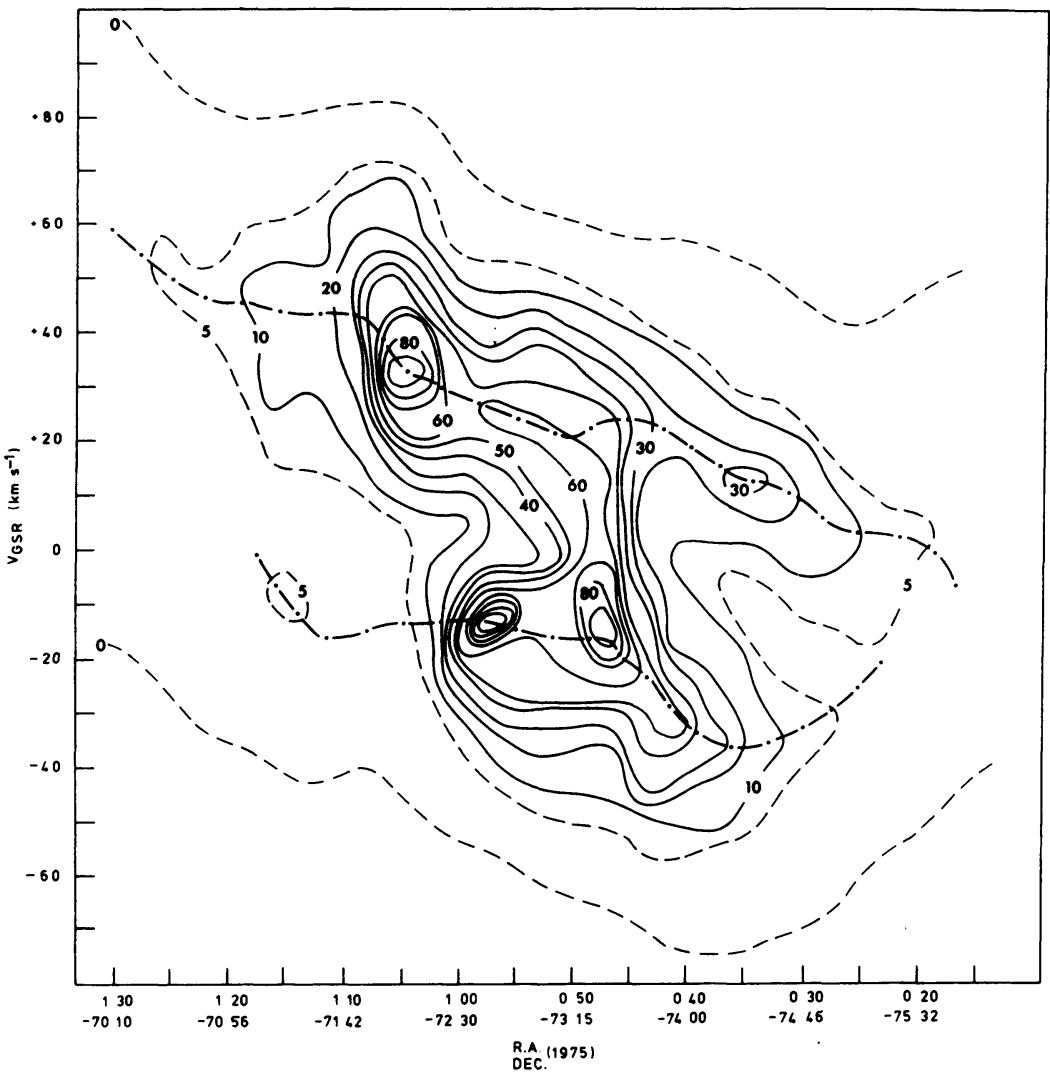

Fig. 4 Radial velocity structure along the major axis of the SMC obtained using the 64-m Parkes radiotelescope. $\mathrm{V}_{\mathrm{GSR}}$ is calculated using $\mathrm{V}_{\mathrm{Q}}=250 \mathrm{~km} \mathrm{~s}^{-1}$. Contour numbers are the brightness temperatures $\left({ }^{\circ}\right)$ of the HI. The dot/dashed lines indicate the ridges of the HI peaks.

Figure 5 shows that stars, HII regions and planetary nebulae also display this dichotomy in their radial velocities. Thus the two HI velocity components in the SMC delineate quite separate entities each with their own nebular and stellar population. Therefore we conclude that the SMC has been badly torn during its near collision with the IMC $2 \times 10^{8}$ years ago (Murai and Fujimoto 1980; Fujimoto 1984) and a large fragment, the Mini-Magellanic Cloud (MMC), is now separating from the remnant of the SMC (SMCR) at about $30 \mathrm{~km} \mathrm{~s}^{-1}$. They are about $6 \mathrm{kpc}$ apart. The CaII absorption line velocities (Feast et al. 1960) are marked along each velocity base-line in Figure 5. They invariably fall under the lower velocity HI profile even though the stars are about equally divided between the two velocity components. Hence the low velocity component must be in front of the high velocity component (Hindman 1964). It is assumed that the lower velocity component is the SMCR as it is the more intense. The SMCR is more compact than the MMC which extends further to the north-east. The red globular clusters 


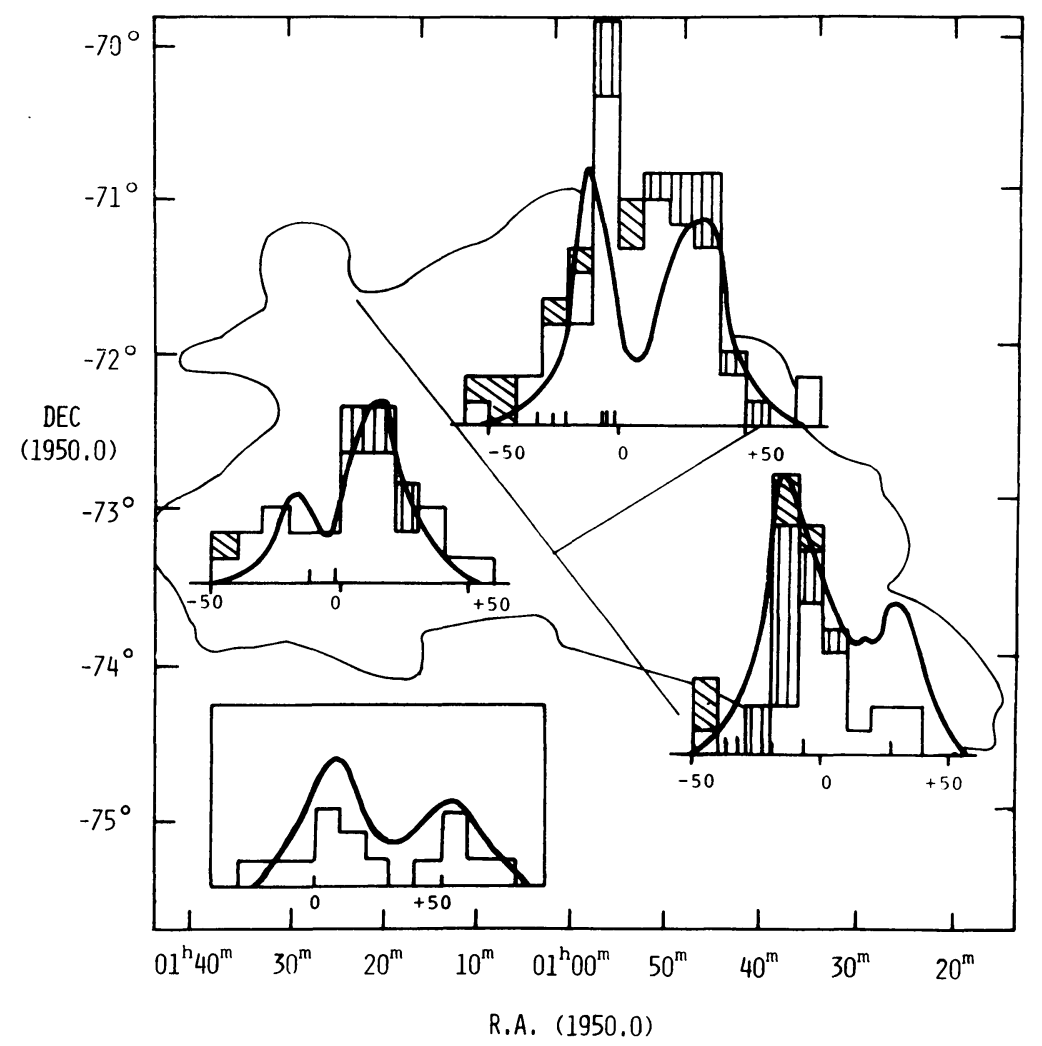

Fig. 5 Synoptic histograms showing the radial velocity distribution $\left(\mathrm{V}_{\mathrm{GSR}}\right)$ of stars (unshaded), HII regions (vertical lines) and planetary nebulae (diagonal lines) drawn on the region of the SMC which they represent indicated by the straight boundary lines. The inset is for the bridge region. ( $V_{G S R}$ is calculated using $\left.V_{\odot}=250 \mathrm{~km} \mathrm{~s}^{-1}\right)$. A HI profile representative of each region is superimposed on each histogram. The CaII absorption line velocities are marked on each velocity baseline. Data taken from Carozzi (1974), Dubois (1975), Feast et al. (1960), Feast (1968), Ardeberg and Maurice (1979), Westerlund and Glaspey (1971), Smith and Weedman (1973), and Hindman (1967).

concentrate around the SMCR at the centre and south end of the Bar whilst the blue clusters extend well to the north-east (Brück 1975).

The splitting of the SMC into two smaller "galaxies" provides a simple explanation for the stellar observations of Ardeberg and Maurice (1979), Florsch et al. (1981) and Welch and Madore (1984) which indicate that the SMC has a large extension along the line of sight. It also explains why the intense regions of $\mathrm{HI}$ as shown in the integrated HI map of Hindman (1967) are displaced with respect to the intense HII regions. Positional agreement becomes good when HI maps of the same velocity as the HII regions are used (MCGee and Newton 1981; Bajaja and Loiseau 1982). 


\section{THE INTER-CLOUD REGION}

The dominating feature of the inter-Cloud region is the intense bridge of gas connecting the SMC with the LMC, which is clear evidence for tidal interaction between the two galaxies. Its HI profiles show the characteristic double peaks separated by $30-40 \mathrm{~km} \mathrm{~s} \mathrm{~s}^{-1}$ of the gas in the SMC (Mathewson et al. 1979). This indicates that both the SMCR and the MMC are the sources of the bridge gas. The velocity maps labelled $-11 \mathrm{~km} \mathrm{~s}^{-1}$ and $+21 \mathrm{~km} \mathrm{~s}^{-1}$ in Figures $\mathrm{la}$ and $\mathrm{lb}$ by Bajaja and Loiseau (1982) show the points of origin in these two "galaxies".

Stars have been identified in the HI condensations in the bridge as far out from the SMC as $3^{\mathrm{h}} 15^{\mathrm{m}}-74^{\circ} 00^{\prime}$. The radial velocities of these stars are divided into similar groups to the gas indicating that they originate in both HI streams (Fig. 5). This stellar component suggests that the bridge is not diffuse halo gas but that each component is probably only a few kpc thick.

The long spurs extending northwards from the bridge towards the Magellanic Stream are a noticeable feature of the inter-cloud region. These spurs must be the result of tidal forces between the IMC and SMC and are the start of the Magellanic Stream. The gas in the inter-cloud region has a mass of $5 \times 10^{8} \mathrm{M}_{\odot}$ and is loosely bound to the Magellanic clouds. Some interaction between halo and Magellanic Cloud gas appears to be taking place as deep $\mathrm{H} \alpha$ photography by Johnson et al. (1982) shows weak, diffuse $H \alpha$ emission (EM 25) between the Magellanic Clouds. In addition, the steep HI gradients along the eastern edge of the IMC and the southern edge of the inter-Cloud region are probably the result of ram pressure of the halo. Mathewson et al. (1977) took this as observational evidence that the direction of the orbit of the Magellanic Clouds is counter-clockwise as viewed from the sun. It is estimated that a gas density of about $10^{-4}$ in the halo of our Galaxy would produce the observed compression of the HI. If the orbital velocity of the Magellanic Clouds is about $300 \mathrm{~km} \mathrm{~s}^{-1}$ and if the masses of the LMC and SMC are $10^{10} \mathrm{M}_{\odot}$ and $2 \times 10^{9} \mathrm{M}_{\odot}$, respectively, a halo gas density of $4 \times 10^{-4}$ would start stripping $\mathrm{HI}$ from the inter-Cloud region.

\section{THE MAGELIANIC STREAM}

Figure 6 shows the HI surface density and velocity contours of MSI obtained using the Parkes $64-\mathrm{m}$ reflector. The narrow elongated structures containing condensations about 30-50 arc min in size with FWHM of 18-25 km s $\mathrm{s}^{-1}$ are characteristic of the Magellanic Stream. Mirabel (1981) has surveyed MSV and MSVI using the Arecibo radio telescope with the very high angular resolution of 3.3 arc min. These observations show much fine structure ( $\sim 5$ arc $\mathrm{min})$ and were interpreted as the ongoing disintegration of the tip of the Magellanic Stream as it approached the Galaxy. The very high negative velocity HI clouds (VHVCs) observed in the first and second quadrants of the southern galactic hemisphere were assumed to be fragments of this disintegration 


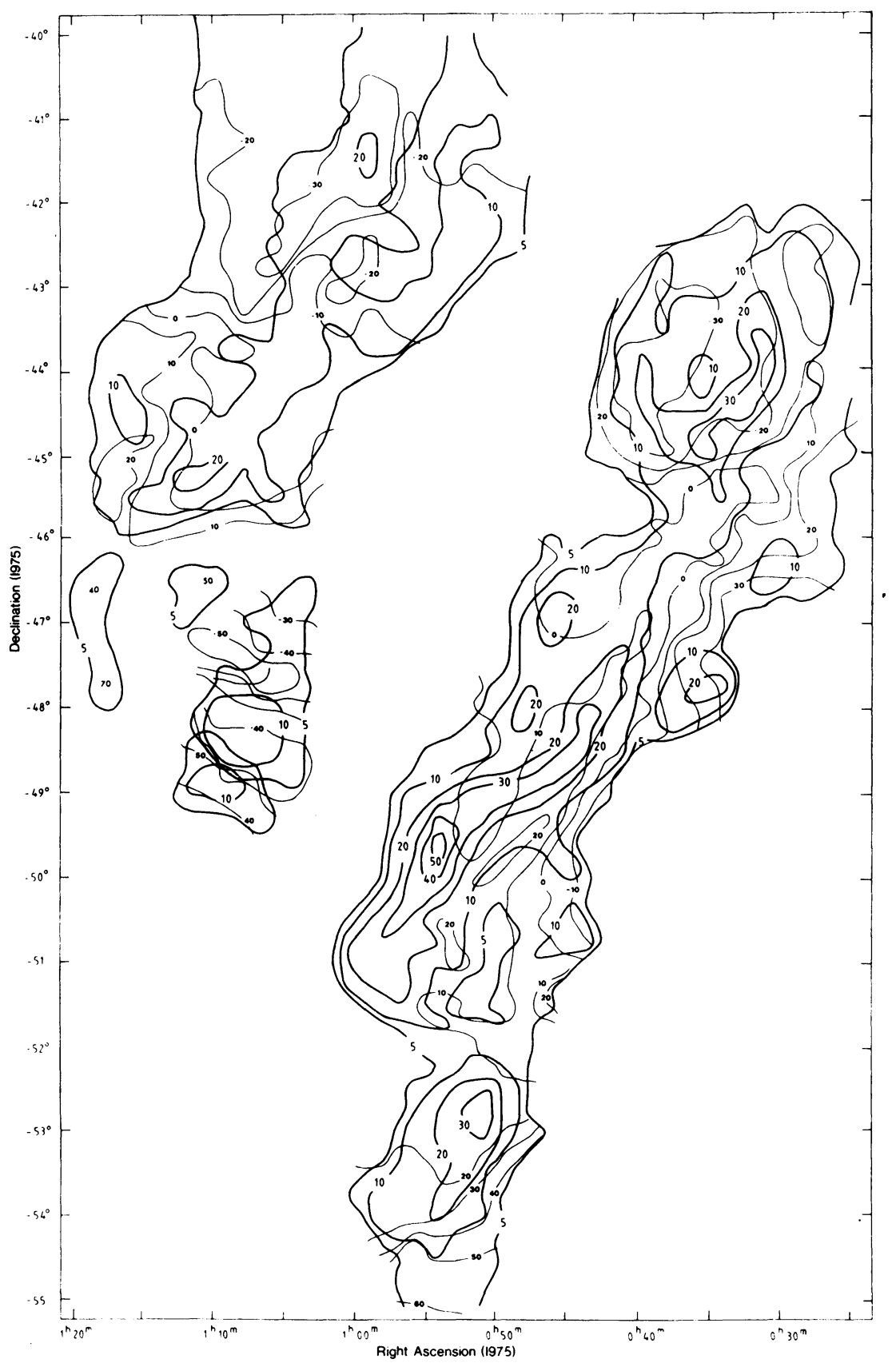

Fig. 6 The thick lines are the HI surface density contours of MSI. The contour unit is $10^{19}$ atoms $\mathrm{cm}^{-2}$. The thin lines are the radial velocity contours. The contour numbers are $V_{G S R}$ in $\mathrm{km} \mathrm{s}^{-1}$ calculated using $\mathrm{V}_{\odot}=225 \mathrm{~km} \mathrm{~s}^{-1}$. Data were obtained using the 64-m Parkes radio telescope. 
scattered over a wide area. Giovanelli (1981) is attracted to this unified theory of HVCs but there are the following difficulties with this currently popular hypothesis:

1) This hypothesis is based on the models of Davies and Wright (1977) and Lin and Lynden-Bell (1977) which place the tip of the Stream 10-15 kpc from the galactic centre. As Lin and Lynden-Bell (1982) have pointed out, these models are incorrect and the tip of the Stream is about $60 \mathrm{kpc}$ from the centre of our Galaxy (Murai and Fujimoto 1980). 2) There are a number of other well-known, unrelated phenomena besides gravitational forces that accelerate HI to high velocities e.g., supernova remnants (Giovanelli and Haynes 1979; Mathewson et al. 1983); gas stripping of galaxies, active galactic nuclei and stellar winds are other examples. The prominent high-latitude galactic radio spurs which have been identified with SNRs, (Berkhuijsen et al. 1971) occupy the first and second quadrants and have the major HVC complexes A, C, and AC (Hulsbosch 1975) lying along their boundaries thus suggesting an association (see also Weaver 1979).

3) The centre of mass of the Local Group of galaxies lies in this region of sky so that intergalactic gas clouds would be expected to lie in these directions. It is likely that some of the VHVCs will be such clouds particularly as a number of intergalactic clouds have now been identified in other groups (Mathewson et al. 1975; Briggs et al. 1980; Hart et al. 1980; Mirabel and Cohen 1979; Schneider et al. 1983). 4) It is not clear how orbits can be generated by the fragmentation of the tip of the Stream which will produce the observed spatial and velocity distribution of the VHVCs, i.e., a fairly uniform $V_{G S R} \sim-250$ $\mathrm{km} \mathrm{s} \mathrm{s}^{-1}$ over $l=0^{\circ}-190^{\circ}$ at low to medium galactic latitudes. 5) MSI-IV may have similar fine structure to MSV and VI but unfortunately the Arecibo reflector cannot observe them.

An example of a group of VHVCs that appear to have a SNR origin is the HI complex near $l=165^{\circ} \mathrm{b}=-45^{\circ}$. Observations by Cohen (1981) show a $25^{\circ}$ long HI streamer at $\mathrm{V}_{\mathrm{LSR}}=-110 \mathrm{~km} \mathrm{~s}^{-1}$ coincident with a -10 $\mathrm{km} \mathrm{s} \mathrm{s}^{-1}$ filament in the local gas at a distance of 100-300 pc. Cohen suggests that the high velocity stream is colliding with the galactic $\mathrm{HI}$ disk and that the $-10 \mathrm{~km} \mathrm{~s} \mathrm{~s}^{-1}$ filament is shocked galactic gas. In a later paper, Cohen (1982) maps a VHVC at $V_{L S R}=-190$ to $-360 \mathrm{~km} \mathrm{~s}^{-1}$ which is the same size as the lower velocity filaments. In fact if Figure 3 of Cohen (1981) is superimposed on Figure 4 of Cohen (1982) the similarity between the shape of the 3 filaments is obvious and the high velocity filament lies along the northern boundary of the lower velocity filaments. The whole complex coincides with the strongest region of radio continuum emission from the Cetus Arc, a nearby SNR (Large et al. 1962). The close spatial relationship between the three HI streams and the SNR almost certainly implies an association and not, as Cohen argues, that the VHVC streamer is debris from the break-up of the tip of the Magellanic Stream. In addition, the intensity of the high velocity component is much greater than MSVI of which it is supposed to be a small fragment. Therefore it is our opinion that the Magellanic stream is as illustrated in Figure 1. 
Murai and Fujimoto (1980) obtained a good fit both to the radial velocities and the spatial structure of the Stream when they introduced their test particles at the beginning of the last orbit of the Magellanic Clouds about $1.8 \times 10^{9}$ years ago. However, when the test particles were introduced $10^{10}$ years ago the fit was poor (Fujimoto 1984). This suggests that the Magellanic Clouds have only made one close passage to our Galaxy in their lifetime. It appears possible to still successfully model the Stream with a hyperbolic orbit providing the massive halo of our Galaxy $\left(10^{12} \mathrm{M}_{\odot}\right)$ used by Murai and Fujimoto is retained.

The concept of a hyperbolic orbit for the Magellanic clouds is attractive for other reasons as well. They are:

1) The damage to the Magellanic Clouds by our massive Galaxy during seven or more close encounters would have been considerable and much more than observed. (Dynamical friction does not introduce a large enough factor to significantly ease the severity of the tidal forces in the earlier passages, Murai and Fujimoto 1980). Whilst the LMC and SMC have tidally interacted, it is the effect of our Galaxy which would remove significant quantities of material from the system. At the moment there is $5 \times 10^{8} \mathrm{M}_{\odot}$ of weakly bound inter-Cloud gas which is about half of the total gas content of the LMC and SMC. It is very likely that this would have been removed long ago if the Magellanic Clouds were bound to the Galaxy. However the gas content of the LMC and SMC is $10 \%$ and $30 \%$ of their total mass, respectively, which is about normal for Magellanic-type galaxies.

2) The morphology of the LMC is archetypal of its class which would be surprising if it has suffered repeated tidal disruption.

3) The hyperbolic orbit explains why the Magellanic clouds and the Stream lie near the plane of the Local Group of galaxies because if the galaxies lie in a plane so must their orbits. The Local Group galaxies (including the Magellanic Clouds) show a systematic variation of radial velocity with angle in the plane of the Local Group which is due to the motion of the Galaxy with respect to the Local Group. The fact that the Magellanic Clouds also reflect this motion supports the conclusion that the Magellanic Clouds are not bound to our Galaxy (Mathewson and Schwarz 1976).

4) The proximity of a galaxy as bright as the LMC $\left(M_{\mathrm{V}} \sim-18.5\right)$ to such a large spiral as our Galaxy is unique which suggests that it is a rare event.

The fact that a group of globular clusters and dwarf spheroidal galaxies with anomalous colour-magnitude diagrams lies close to the orbital plane of the Magellanic Clouds is interpreted by Lynden-Bell (1976) and Kunkel (1979) as relics of tidal interaction between the Magellanic Clouds and the Galaxy on the occasion of an early encounter. However in the context of our present scenario, they would need to be the result of the merger of another galaxy with our Galaxy some $7 \times 10^{9}$ years ago. Schweizer et al. (1983) point out that these near polar rings are favored statistically because of their slow differential 
precession and consequent longevity; alternatively if the halo of our Galaxy is triaxial, some orbits would tend to migrate toward the poles. Their Figure 2 shows examples of polar rings around galaxies which may be the result of mergers.

\section{REFERENCES}

Ardeberg, A., and Maurice, E.: 1979, Astron. Astrophys. 77, 277. Bajaja, E., and Loiseau, N.: 1982, Astron. Astrophys. Suppl. 48, 71. Berkhuijsen, E., Haslam, C.G.T., and Salter, C.J.: 1971, Astron. Astrurhys. 14, 252 .

Briggs, F.H., Wolfe, A.M., Krumm, N., and Salpeter, E.E.: 1980, Astrophys. J., 238, 510.

Brück, M.T.: 1975, Mon. Not. Roy. astr. Soc. 173, 327.

Carozzi, N.: 1974, Astron. Astrophys. Suppl. 16, 277.

Cohen, R.J.: 1981, Mon. Not. Roy. astr. Soc. 196, 835.

Cohen, R.J.: 1982, Mon. Not. Roy. astr. Soc. 199, 281.

Cohen, R.J.: 1982, Mon. Not. Roy. astr. Soc. 200, 391.

Davies, R.D., and Wright, A.E.: 1977, Mon: Not. Roy. astr. Soc. 180, 71. Dubois, P.: 1975, Astron. Astrophys. 40, 227.

Feast, M.W.: 1968, Mon. Not. Roy. astr. Soc. 140, 345.

Feast, M.W., Thackeray, A.D., and Wesselink, A.J.: 1960, Mon. Not. Roy. astr. Soc. $121,337$.

Feitzinger, J.V.: 1980, Space Sci. Rev. 27, 35.

Feitzinger, J.V., Isserstedt, J., and Schmidt-Kaler, Th.: 1977, Astron. Astrophys. 57, 265.

Florsch, A., Marcout, J., and Fleck, E.: 1981, Astron. Astrophys. $96,158$.

Fujimoto, and Murai, T.: 1984, this volume, p. 115.

Giovanelli, R.: 1981, Astron. J. 86, 1468.

Giovanelli, R., and Haynes, M.P.: 1979, Astrophys. J. 230, 404.

Hart, L., Davies, R.D., and Johnson, S.C.: 1980, Mon. Not. Roy. astr. Soc. 191, 269.

Haynes, M.P.: 1979, Astron. J. $84,1173$.

Hindman, J.V.: 1964, Nature, 202, 377.

Hindman, J.V.: 1967, Aust. J. Phys. 20, 147.

Hulsbosch, A.N.M.: 1975, Astron. Astrophys. 40, 1.

Johnson, P.G., Meaburn, J., and Osman, A.M.I.: 1982, Mon. Not. Roy. astr. Soc. 198, 985.

Kunkel, W.E.: 1979, Astrophys. J., 228, 718.

Large, M.I., Quigley, M.J.S., and Haslam, C.G.T.: 1962, Mon. Not. Roy. astr. Soc. 124, 405.

Lin, D.N.C., and Lynden-Bell, D.: 1977, Mon. Not. Roy. astr. Soc. 181, 37.

Lin, D.N.C., and Lynden-Bell, D.: 1982, Mon. Not. Roy. astr. Soc. 198, 707.

Lynden-Bell, D.: 1976, Mon. Not. Roy. astr. Soc. 174, 695.

McGee, R.X., and Milton, J.A.: 1966, Aust. J. Phys. 19, 343.

McGee, R.X., and Newton, L.M.: 1981, Proceedings of the Astronomical Society of Australia, 4, 189. 
Mathewson, D.S.: 1976a, Proceedings of the Astronomical Society of Australia, 3, 20.

Mathewson, D.S.: 1976b, Roy. Greenwich Obs. Bull. No. 182, 217.

Mathewson, D.S., Cleary, M.N., and Murray, J.D.: 1975, Astrophys. J. (Letters), 195, L97.

Mathewson, D.S., Ford, V.L., Dopita, M.A., Tuohy, I.R., Long, K.S., and Helfand, D.J.: 1983, Supernova Remnants and Their X-ray Emission, IAU Symp. \#101, ed. J. Danziger and P. Gorenstein, (Dordrecht: Reidel).

Mathewson, D.S., Ford, V.L., and Fisher, J.L.: 1983 (in preparation). Mathewson, D.S., Ford, V.I., Schwarz, M.P., and Murray, J.D.: 1979, The Large Scale Characteristics of the Galaxy, IAU Symp. \#84, ed. W. B. Burton, (Dordrecht: Reidel), p. 547.

Mathewson, D.S., and Schwarz, M.P.: 1976, Mon. Not. Roy. astr. Soc. 176, 47.

Mathewson, D.S., Schwarz, M.P., and Murray, J.D.: 1977, Astrophys. J. (Letters), 217, L5.

Mirabel, I.F.: 1981, Astrophys. J. 250, 528.

Mirabel, I.F.: 1982, Astron. J. 256, 120.

Mirabel, I.F., and Cohen, R.J.: 1979, Mon. Not. Roy. astr. Soc., $188,219$.

Morras, R.: 1983, Astron. J. 88, 62.

Murai, T., and Fujimoto, M.: 1980, Publ. astr. Soc. Japan, 32, 581.

Rogstad, D.H., Lockhart, I.A., and Wright, M.C.H.: 1974, Astrophys. J. $193,309$.

Rohlfs, K., Feitzinger, J.V., and Kreitschmann, J.: 1984, this volume, p. 395.

Schneider, S.E., Helou, G., Salpeter, E.E., and Terzian, Y.: 1983, Astrophys. J. (in press).

Schweizer, F., Whitmore, B.C., and Rubin, V.C.: 1983, Astron. J. 88, 909.

Smith, M.G., and Weedman, D.W.: 1973, Astrophys. J., 179, 461.

Wannier, P., and Wrixon, G.T.: 1972, Astrophys. J. (Letters), 173, L119.

Weaver, H.: 1979, The Large Scale Characteristics of the Galaxy, IAU

Symp. No. 84, ed. W. B. Burton, (Dordrecht: Reidel), 295.

Welch, D.L., and Madore, B.F.: 1984, this volume, p. 221.

Westerlund, B.E., and Glaspey, J.: 1971, Astron. Astrophys. 10, 1.

\section{DISCUSSIOR}

Fall: Since your two components of the SMC are seen in projection, the line of sight; velocity of $30-40 \mathrm{~km} / \mathrm{s}$ must be close to the true velocity of separation. At the relative separataion of a few kpc, the pair of SMC comporients are probably bound i.e. not escaping from each other. Thus they will probably collapse back together and nerge in 1 Gyr or less. Should we really think of the SilC as two galaxies in this concept?

Mathewson: The escape velocity of the Mini Magellanic Cloud from the remart of the imall wagellanic Cloud is about, $35 \mathrm{~km} / \mathrm{s}$ which is about their observed separation velocity. Therefore they may never coalesce. 